\title{
The approach to calculate the aerodynamic drag of maglev train in the evacuated tube
}

\author{
Jiaqing Ma $\cdot$ Dajing Zhou $\cdot$ Lifeng Zhao \\ Yong Zhang $\cdot$ Yong Zhao
}

Received: 7 July 2013/Revised: 28 July 2013 / Accepted: 11 August 2013/Published online: 13 September 2013

(C) The Author(s) 2013. This article is published with open access at Springerlink.com

\begin{abstract}
In order to study the relationships between the aerodynamic drag of maglev and other factors in the evacuated tube, the formula of aerodynamic drag was deduced based on the basic equations of aerodynamics and then the calculated result was confirmed at a low speed on an experimental system developed by Superconductivity and New Energy R\&D Center of South Jiaotong University. With regard to this system a high temperature superconducting magnetic levitation vehicle was motivated by a linear induction motor (LIM) fixed on the permanent magnetic guideway. When the vehicle reached an expected speed, the LIM was stopped. Then the damped speed was recorded and used to calculate the experimental drag. The two results show the approximately same relationship between the aerodynamic drag on the maglev and the other factors such as the pressure in the tube, the velocity of the maglev and the blockage ratio. Thus, the pressure, the velocity, and the blockage ratio are viewed as the three important factors that contribute to the energy loss in the evacuated tube transportation.
\end{abstract}

Keywords Evacuated tube - Maglev train · Aerodynamic drag . Pressure in the tube

J. Ma · D. Zhou $\cdot$ L. Zhao · Y. Zhang $\cdot$ Y. Zhao Superconductivity and New Energy R\&D Center, Southwest Jiaotong University, Chengdu 610031, Sichuan, China

J. Ma $(\bowtie) \cdot$ D. Zhou $\cdot$ L. Zhao · Y. Zhang $\cdot$ Y. Zhao Key Laboratory of Magnetic Levitation Technologies and Maglev Trains (Ministry of Education of China), Southwest Jiaotong University, Chengdu 610031, Sichuan, China e-mail:357287962@qq.com

Y. Zhao

School of Materials Science and Engineering, University of New South Wales, Sydney, NSW 2052, Australia

\section{Introduction}

The speed of traditional trains is limited because of the dynamic friction between the wheels of the train and the fixed rail on the ground. When the trains are running at a low speed, most of the energy is consumed by friction. The trains can be levitated above the rail to avoid such friction with the technology of magnetic levitation [1]. There are three types of levitation technologies: electromagnetic suspension (EMS), electrodynamic suspension (EDS), hybrid electromagnetic suspension (HEMS) [2]. Even with these three methods, the velocity of trains could not be improved remarkably because of the aerodynamic drag. When the trains run at low speeds, this drag is not evident. At high speeds, however, the aerodynamic drag is too large to be neglected. Whatever the trains are levitated or not, the aerodynamic drag is the dominate part of drag when it runs at a high speed in the atmosphere near the earth's surface. At a speed range between 400 and $500 \mathrm{~km} / \mathrm{h}$, the aerodynamic drag accounts for $80 \%-90 \%$ of the total drag including the aerodynamic drag, the eddy resistance force, and the braking force [3]. The train speed is much lower than the airplane speed because airplanes flight in a circumstance of rarefied gas in the high altitude. In view of this fact, the evacuated tube transportation (ETT) was proposed to reduce the aerodynamic drag and improve the speed of the maglev train. Shen [4] and Yan [5] discussed the possibility, strategy, and the technical proposal for developing the ETT in China.

Theoretically speaking, when the inner part of the tube is in the condition of absolute vacuum, the aerodynamic drag for the levitation train inside the tube will be zero. However, this is very hard to realize. An alternative is to draw-off the gas partly and optimize the train shape. Therefore, the influence of the air pressure, the velocity, and the blockage ratio on the Maglev train in the evacuated 
tube system is a very interesting topic to study. Up to now, some research works have been done to explore what conditions are suitable for future ETT. Raghuathan and Kim [6] reviewed the state of the art on the aerodynamic and aeroacoustic problems of high-speed railway train and highlighted proper control strategies to alleviate undesirable aerodynamic problems of high-speed railway system. Various aspects of the dynamic characteristics were reviewed and aerodynamic loads were considered to study the aerodynamic drag [7]. Wu et al. [8] simulated the maglev train numerically with software STARCD based on the $\mathrm{N}-\mathrm{S}$ equation of compressible viscosity fluid and $\mathrm{k}-\varepsilon$ turbulence model. The flow field, the pressure distribution and the aerodynamic drag coefficient were also analyzed to illustrate the relationship between the aerodynamic drag and the shape of the train in evacuated tube. The pressure distribution in the whole flow field and the relation between the aerodynamic drag and the basic parameters were derived in [9]. Shu et al. [10] simulated the flow field around the train based on the 3D compressible viscous fluid theory and draw the conclusion that its aerodynamic performance is relevant to the length of the streamlined nose. In [11-13], the simulated results showed that the speed, the pressure and the blockage ratio significantly affect the aerodynamic drag of the train in an evacuated tube.

In this paper, the experimental system model developed by Superconductivity and New Energy R\&D Center of South Jiaotong University was used to study the aerodynamic drag in the tube. The basic mass conversation equation and the momentum conversation equation [14] were used at first to deduce the expression that describes the relationships between the drag and the main parameters such as the tube pressure, velocity, and the blockage ratio. Then the aerodynamic drag is calculated with that expression. Finally, the calculated results are compared with the experimental data to verify the validity of the deduced expression.

\section{The mathematical model of the aerodynamic drag and the physical model of the ETT}

In this paper, the $\mathrm{N}-\mathrm{S}$ equation of compressible viscosity fluid and $\mathrm{k}-\varepsilon$ turbulence model are applied to calculating the flowing field of aerodynamics. Considering an infinitesimal part for any arbitrary circumstance, it is well known that every part follows the laws of mass conversation equation and the momentum conversation equation. These two equations $[15,16]$ are listed as:

$$
\begin{aligned}
& \frac{\partial \rho}{\partial t}+\nabla \cdot(\rho v)=0, \\
& \frac{\partial\left(\rho v_{\mathrm{i}}\right)}{\partial t}+\nabla \cdot\left(\rho v_{\mathrm{i}} v\right)=\nabla \cdot\left(\mu \nabla v_{\mathrm{i}}\right)-\frac{\partial p}{\partial x}+F_{\mathrm{i}},
\end{aligned}
$$

where

$\rho=\rho(x, y, z, t)$

$\rho$ is density of the infinitesimal part, $v$ velocity of the infinitesimal part, $v_{i}$ each component of velocity (different when in different coordinates), $F_{i}$ body force in each direction, $\mu$ dynamic viscosity, and $p$ pressure of the infinitesimal part.

Eq. (1) means that the quality flowing into the infinitesimal part is equal to that out of this part. And Eq. (2) means that the rate of change of arbitrary mass's momentum is equal to the sum of the force acting upon it.

All of the following mathematical derivations in this paper are based on Eqs. (1) and (2). To study the evacuated tube transportation, an experimental model was developed.

This model called evacuated tube system for maglev train (ETSMT) includes three components: the evacuate tube, maglev, and propulsion system.

The tube is made of Perspex with the circumference of $10 \mathrm{~m}$ and the circular permanent magnetic guideway (PMG) is placed along the bottom of the tube. The positions of the tube, the train, and the PMG and the used rectangular coordinate system are shown in Fig. 1.

In Fig. $1, a_{0}$ and $b_{0}$ stand for the width and the height of the tube respectively; $a, b$, and $c$ stand for the width, the height, and the length of the maglev train, respectively. $h_{0}$ is the height of the PMG and $h$ the levitated height.

As shown in Fig. 1, the train is levitated above the rail with height $h$ and can only move in the $x$-direction due to the selfguiding characters of high-temperature-superconducting (HTS)-PMG system. For convenience, we suppose:

1) The tube is straight and the train runs along it only in the $x$-direction.

2) The magnetic flux of permanent magnetic rail is constant in the $x$-direction.

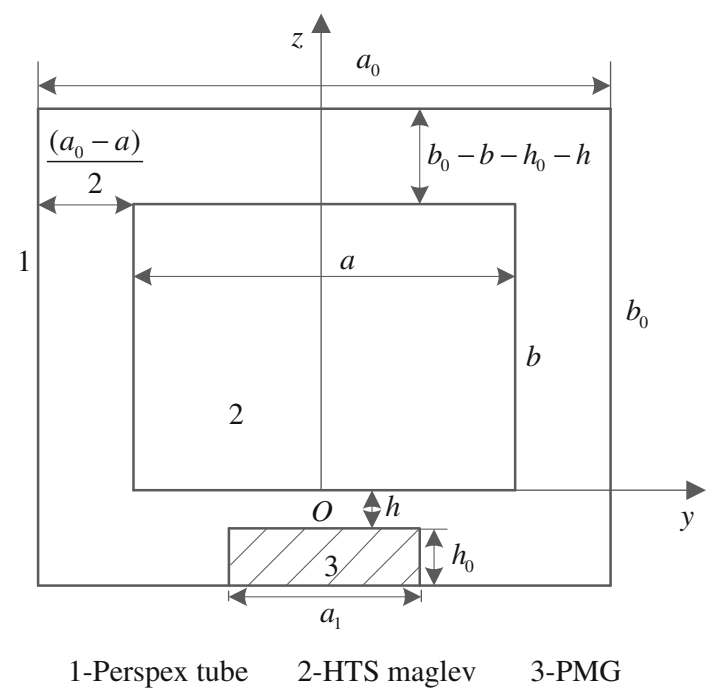

Fig. 1 Schematic diagram of $y z$-section of ETTSMT 
3) The pressure of any part of inner tube is constant in the $z$-direction with neglecting the atmospheric molecular mass.

4) The train is in the center of the tube whatever the blockage rate is.

In the Cartesian rectangular coordinate system the velocity is expressed as:

$v=v_{\mathrm{x}} e_{\mathrm{x}}+v_{\mathrm{y}} e_{\mathrm{y}}+v_{\mathrm{z}} e_{\mathrm{z}}$

where $\rho$ is the atmospheric density, $v_{\mathrm{x}}, v_{\mathrm{y}}, v_{\mathrm{z}}$ are the components of velocity in $x, y, z$ axis directions respectively, $e_{\mathrm{x}}, e_{\mathrm{y}}, e_{\mathrm{z}}$ are the unit vectors of each axis. When considering the assumptions of (1) and (2), $v_{\mathrm{y}}$ and $v_{\mathrm{z}}$ are zero. Then Eqs. (1) and (2) are modified as:

$\frac{\partial \rho(x, t)}{\partial t}+\nabla \cdot\left(\rho v_{\mathrm{x}} e_{\mathrm{x}}\right)=0$,

$\frac{\partial\left[\rho(x, t) v_{\mathrm{x}}\right]}{\partial t}+\nabla \cdot\left[\rho(x t) v_{\mathrm{x}}^{2} \boldsymbol{e}_{\mathrm{x}}\right]=\nabla \cdot\left(\mu \nabla v_{\mathrm{x}}\right)-\frac{\partial \rho}{\partial x}+F_{\mathrm{x}}$.

To demonstrate the evident effect of the pressure in tube, the streamlined nose of the train is not adopted. The schematic diagram in moving direction of train is shown in Fig. 2.

As shown in Fig. 2, the maglev train is levitated above the PMG and there is no dynamic friction between the train and the rail. According to assumption (2), there is no vibration in the $z$-direction, which means that all of the kinetic energy of the free levitated running train is consumed because of the aerodynamic drag after the train gains the initial kinetic energy. In an ideal situation, when the tube pressure is zero, the aerodynamic drag is equal to zero. Since this condition is almost impossible to realize, the actual practice is to pull the air out of the tube to form a suitable pressure. The purpose of this work is to explore the relationship between the drag and the tube pressure.

The aerodynamic drag of a running maglev in this system is composed of three components:

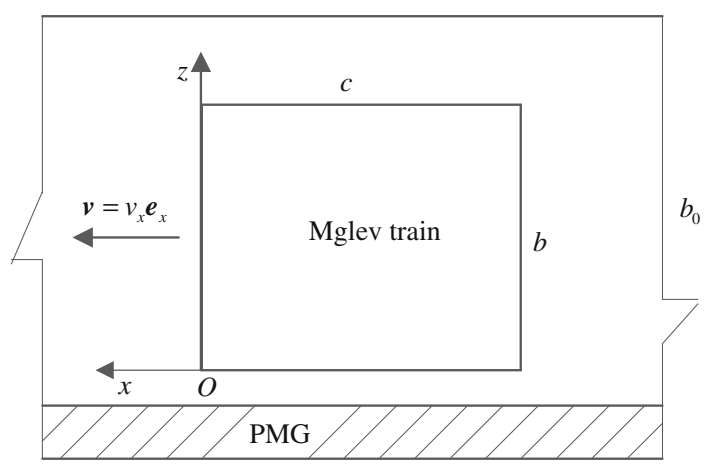

Fig. 2 The schematic diagram of xz-section of ETTSMT
(1) $F_{1}$ : the force on windward side of the train due to the collision between air and the train,

(2) $F_{2}$ : the air friction on four side faces of the train and

(3) $F_{3}$ : the force caused by the different pressures of windward side and the tailstock side of the train

Each force will be discussed in following sections based on Eqs. (4) and (5).

\subsection{The calculation of $F_{1}$}

For simplification, a long section of the air ahead of the train is moving at the same velocity as the train because of the character of the air. An infinitesimal part of the air in area 1 is considered in Fig. 3. We suppose that the velocity of the thin layer of air is to vary after a tiny time $\mathrm{d} t$ after collision and the displacement of this layer is $\mathrm{d} x$ away from the windward side along $x$-axis within another tiny time $\mathrm{d} t$.

The velocity of the infinitesimal air before collision is $v_{1}=0$.

After collision, its velocity is equal to that of the train's. So the kinetic energy of this air is $\frac{1}{2} \rho \mathrm{d} x \mathrm{~d} y \mathrm{~d} z \cdot v_{\mathrm{x}}^{2}$ and we have the equation:

$\frac{1}{2} \rho \mathrm{d} x \mathrm{~d} y \mathrm{~d} z \cdot v_{\mathrm{x}}^{2}=d F_{1 \mathrm{x}} d x$.

$F_{1}$ is equal to zero when the velocity of the train is smaller than sound velocity because the velocity of atmospheric molecule is equal to the sound velocity after the collision with the windward side of the train. When the velocity of train is larger than sound velocity, the air column with the length of $v_{\mathrm{c}} \cdot \mathrm{d} t$ is affected within the period of $\mathrm{d} t$ and the velocity of that air column is approximatively equal to train velocity. The force can be calculated by combining Eqs. (6) and (7) and the momentum conversation equation:

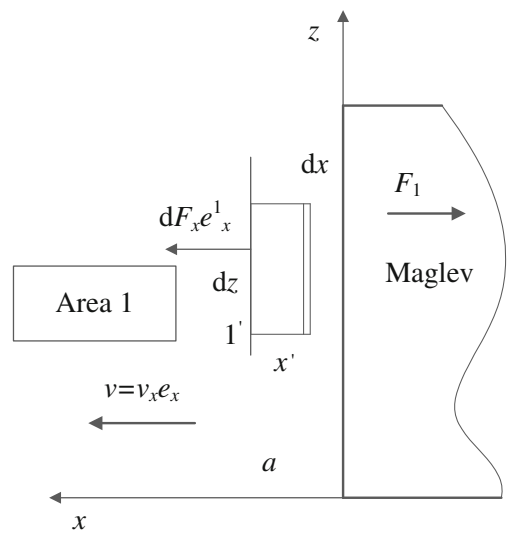

Fig. 3 Schematic diagram of the $F_{1}$ 


$$
\begin{array}{r}
F_{1}=-e_{\mathrm{x}} \iint_{\mathrm{yz}} \mathrm{d} F_{2 \mathrm{x}}=-e_{\mathrm{x}} \iint_{\mathrm{yz}} \frac{1}{2} \rho_{0} v_{\mathrm{x}}^{2} \mathrm{~d} y \mathrm{~d} z \\
=-e_{\mathrm{x}} \frac{p}{2 p_{0}} \rho_{0} v_{\mathrm{c}} \iint_{\mathrm{yz}} v_{\mathrm{x}} \mathrm{d} y \mathrm{~d} z
\end{array}
$$

Thus,

$F_{1}= \begin{cases}0, & v_{\mathrm{x}}<v_{\mathrm{c}} \\ -e_{\mathrm{x}} v_{\mathrm{c}} \iint_{\mathrm{yz}} \frac{p}{2 p_{0}} \rho_{0} v_{\mathrm{x}} d y d z, & \\ v_{\mathrm{x}} \geq v_{\mathrm{c}}\end{cases}$

where $p_{0}=101,325 \mathrm{~Pa}$, and $\rho_{0}=1.293 \mathrm{~kg} / \mathrm{m}^{3}$.

Because of the Brownian movement of molecules, it is reasonable to neglect $F_{1}$ when the train runs at a low speed.

\subsection{The calculation of $F_{2}$}

In this EETSMT, there are four side faces where friction force generates, as shown in Fig. 4, where $F_{2 \mathrm{U}}, F_{2 \mathrm{~B}}, F_{2 \mathrm{~L}}$ and $F_{2 \mathrm{R}}$ represent the frictions on upper side, lower side, left side, and right side respectively. The left side is toward the inside of the paper and the right side is toward the outside.

On upper side, considering a infinitesimal volume of $\mathrm{d} x \mathrm{~d} y \mathrm{~d} z$, the area of contact between the infinitesimal and the air is $\mathrm{d} s=\mathrm{d} x \mathrm{~d} y$.

This component of the aerodynamic drag $F_{2}$ exits because of gas viscidity. The regularity of the fluid velocity distribution between the tube wall and train body side is described by the function of variable $z$ [16]:

$v_{\mathrm{x}}^{\prime}=f_{2 \mathrm{U}}(n) \quad\left(b \leq n \leq b_{0}\right)$,

where $f_{2 \mathrm{U}}$ is velocity function of length $n ; b$ and $b_{0}$ are shown in Fig. 1.

The relationship between the fluid internal friction stress and the velocity gradient according to Newton's proposal is

$\tau=\mu \frac{\partial f_{2 \mathrm{U}}(n)}{\partial n}$,

where $\tau$ friction stress, $\mu$ viscosity,

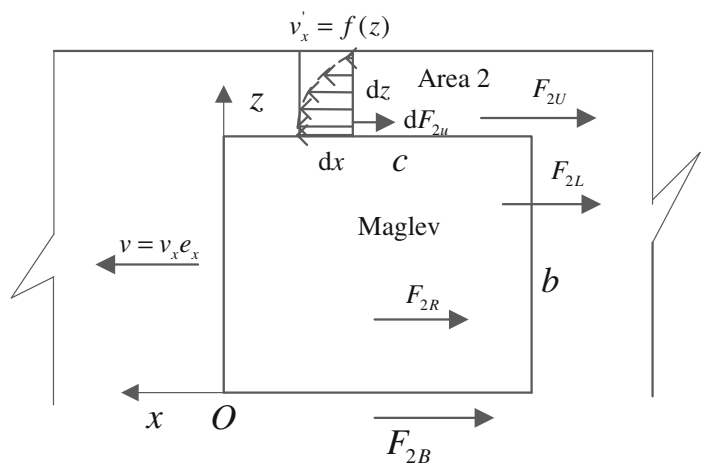

Fig. 4 Schematic diagram of the $F_{2}$ $\frac{\partial f_{2 \mathrm{U}}(n)}{\partial n}$ is the change rate of the velocity from train body side to the tube wall. The friction stress is the force on a unit area with the direction perpendicular to the velocity, and the viscosity $\mu$ is affected by the temperature instead of the air pressure. So the $\mu$ is constant when the temperature is unchanged. From the analysis above, the friction of infinitesimal $\mathrm{d} x \mathrm{~d} y \mathrm{~d} z$ is

$\mathrm{d} F_{2 \mathrm{U}}=\tau \mathrm{d} y \mathrm{~d} x$

$\boldsymbol{F}_{2 \mathrm{U}}=-\boldsymbol{e}_{\mathrm{x}} \mu \iint_{S_{2 \mathrm{U}}} \frac{\partial f_{\mathrm{U}}(n)}{\partial n} \mathrm{~d} x \mathrm{~d} y$.

Likewise, $F_{2 \mathrm{~B}}, F_{2 \mathrm{~L}}$ and $F_{2 \mathrm{R}}$ can be deduced.

Then $F_{2}=F_{2 \mathrm{U}}+F_{2 \mathrm{~B}}+F_{2 \mathrm{~L}}+F_{2 \mathrm{R}}$

$$
\begin{gathered}
=-e_{\mathrm{x}} \mu\left[\iint_{S_{2 \mathrm{U}}} \frac{\partial f_{\mathrm{U}}(n)}{\partial n} \mathrm{~d} x \mathrm{~d} y+\iint_{S_{2 \mathrm{~B}}} \frac{\partial f_{\mathrm{B}}(n)}{\partial n} \mathrm{~d} x \mathrm{~d} y\right. \\
\left.+\iint_{S_{2 \mathrm{~L}}} \frac{\partial f_{\mathrm{L}}(n)}{\partial n} \mathrm{~d} x \mathrm{~d} z+\iint_{S_{2 \mathrm{R}}} \frac{\partial f_{\mathrm{R}}(n)}{\partial n} \mathrm{~d} x \mathrm{~d} z\right]
\end{gathered}
$$

2.3 The calculation of $F_{3}$

Figure 5 shows that $F_{3}$ is generated by pressure difference between the headstock and the tailstock side of the train. This force is larger when the velocity of the train is greater.

In Fig. 5, the pressure of the inner tube is $p$. The train windward side is $x-z$ side with area $S$. For a small time interval $d t$, the train moves with distance $d x$. And there is no interpenetration of air between different areas 1,2 , and 3 within $\mathrm{d} t$. So the velocity of infinitesimal gas is $v_{\mathrm{x}}$ when taking the train as a reference. According to the Bernoulli formula, the relationship between the pressure and the velocity at point $A$ is:

$p_{31}=p+\frac{\rho}{2} v_{\mathrm{x}}^{2}$

And the pressure at point $B$ is

$p_{32}=p$

Fig. 5 Schematic diagram of the $F_{3}$ 
So the pressure difference is:

$F_{3}=-e_{\mathrm{x}} \iint_{\mathrm{yz}} \frac{\rho}{2} v_{\mathrm{x}}^{2} \mathrm{~d} y \mathrm{~d} z$.

2.4 Approximation of the total aerodynamic drag

The blockage rate is defined as

$b_{\mathrm{r}}=\frac{a \cdot b}{a_{0} \cdot b_{0}}=\frac{S}{S_{0}}$,

where $a, b, a_{0}$ and $b_{0}$ are also illustrated in Fig. 1.

When the train is running, the pressure, density, and flow velocity of arbitrary gas are functions of time and space. According to the assumptions and definitions mentioned above, Eqs. (9), (14), and (17) could be modified as:

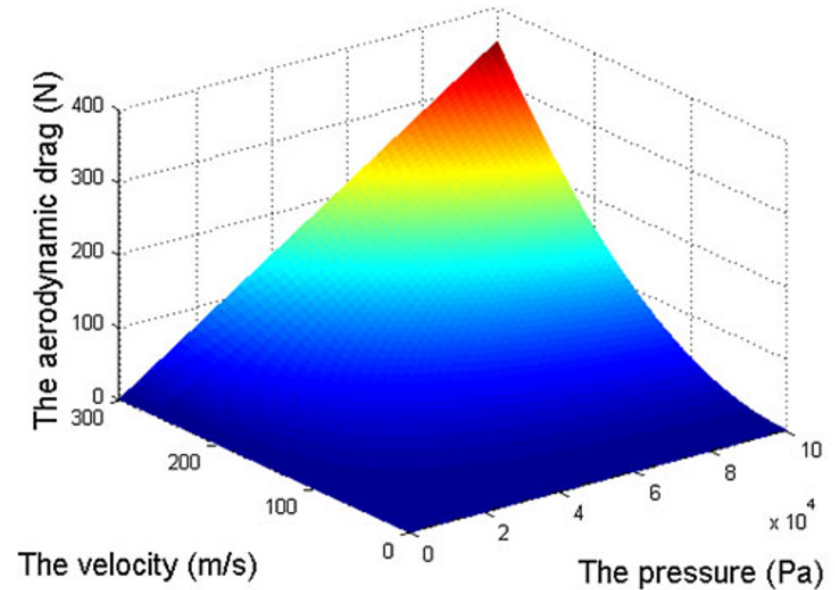

(a) $b_{r}=0.1$

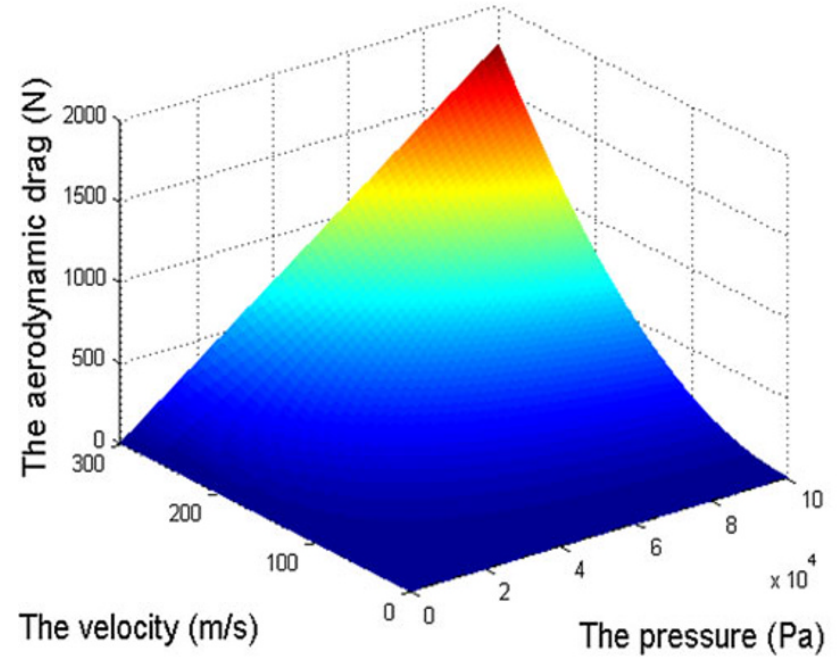

(c) $b_{r}=0.5$

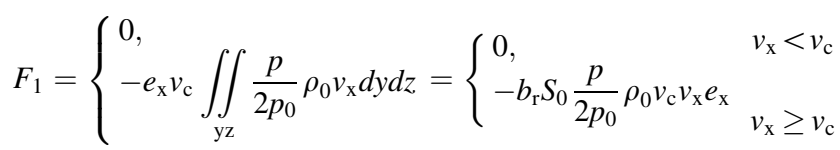

$$
\begin{aligned}
& F_{2}=-\boldsymbol{e}_{x}\left[\iint_{S_{2 U}} \mu \frac{\partial f_{U}(n)}{\partial n} d x d y+\iint_{S_{2 B}} \mu \frac{\partial f_{B}(n)}{\partial n} d x d y\right. \\
&\left.+\iint_{S_{2 L}} \mu \frac{\partial f_{L}(n)}{\partial n} d x d z+\iint_{S_{2 R}} \mu \frac{\partial f_{R}(n)}{\partial n} d x d z\right] \\
&=- \boldsymbol{e}_{x} \mu c v_{x}\left(\frac{a}{b_{0}-b-h_{0}-h}+\frac{a}{h+h_{0}}+\frac{4 b}{a_{0}-a}\right) \\
&=-\boldsymbol{e}_{x} \mu c v_{x} \sqrt{S_{0} b_{r}} .
\end{aligned}
$$

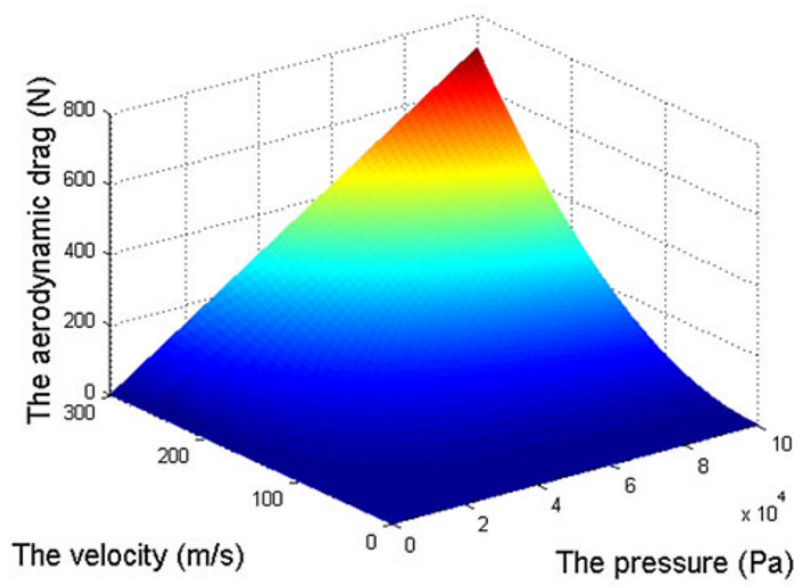

(b) $b_{r}=0.2$

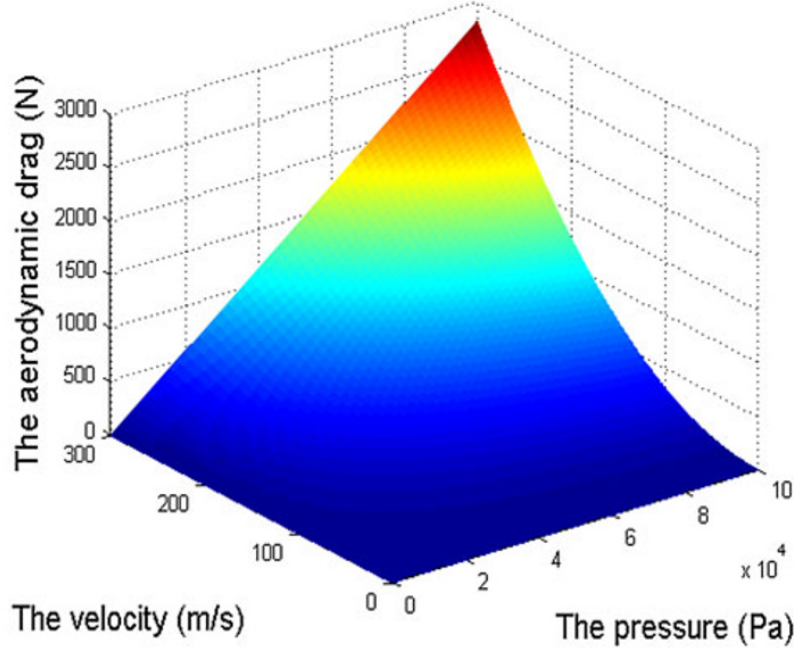

(d) $b_{r}=0.8$

Fig. 6 The total calculated drag when blockage rate is $0.1,0.2,0.5,0.8$, respectively 


$$
\begin{aligned}
F_{3} & =-e_{\mathrm{x}} \iint_{\mathrm{yz}}\left(0+\frac{\rho}{2} v_{\mathrm{x}}^{2}\right) d y d z \\
& =-e_{\mathrm{x}} \int_{-a / 2}^{a / 2} \int_{0}^{b}\left(0+\frac{p \rho_{0}}{2 p_{0}} v_{\mathrm{x}}^{2}\right) d y d z=-e_{\mathrm{x}} b_{\mathrm{r}} S_{0} \frac{p \rho_{0}}{2 p_{0}} v_{\mathrm{x}}^{2}
\end{aligned}
$$

The total aerodynamic drag is expressed as

$F_{\mathrm{x}}\left(b_{\mathrm{r}}, v_{\mathrm{x}}, p\right)= \begin{cases}b_{\mathrm{r}} S_{0} \frac{p}{2 p_{0}} \rho_{0} v_{\mathrm{x}}^{2}+\mu c v_{\mathrm{x}} \sqrt{S_{0} b_{\mathrm{r}}}, & v_{\mathrm{x}}<v_{\mathrm{c}}, \\ b_{\mathrm{r}} S_{0} \frac{p}{2 p_{0}} \rho_{0} v_{\mathrm{x}}\left(v_{\mathrm{x}}+v_{\mathrm{c}}\right)+\mu c v_{\mathrm{x}} \sqrt{S_{0} b_{\mathrm{r}}}, & v_{\mathrm{x}} \geq v_{\mathrm{c}} .\end{cases}$

According to Eq. (22), the relations between the total drag and the blockage ratio, the velocity and the pressure are shown in Fig. 6.

According to Fig. 6, we can calculate the total aerodynamic drag with Eq. (22) and the known parameters of blockage ratio $b_{r}$, velocity $v$, and pressure $p$, and easily obtain the relationship between them.

\section{The experimental system of the evacuated tube}

Figure 7 shows the ETSMT located in a tube made up of Perspex. It is vacuumized with a vacuum pump and the pressure inside the pipe can be detected by an instrument. We designed a control system to gain a fixed pressure ranging from 2,000 to 101,325 $\mathrm{Pa}$. The experimental steps are listed:

1) The HTS maglev was fixed above the PMG with nonferromagnetic material at some height such as $0.01 \mathrm{~m}$ and then the liquid nitrogen was poured into the train. After the train was levitated, the non-ferromagnetic material must be removed from the PMG.

2) The opening hole of the pipe was covered and then the vacuum pump was started with the control system to reach the design pressures such as $10,000,8,000$, and $5,000 \mathrm{~Pa}$ and etc.

3) The liner induction motor was started and then the train could be drove to move when the maglev train runs near the LIM. Thus, the train speed can be accelerated to a certain value such as $3 \mathrm{~m} / \mathrm{s}$.

4) The LIM was stopped when the train's speed reached an expected value. Then the time difference between the position check points $A$ and $B$ was recorded to gain the decreasing train velocity.

5) The velocity was calculated with necessary parameters.

All parameters of this experimental system in Fig. 1 are listed in Tables 1 and 2.
When $T=288.15 \mathrm{~K}, \mu=1.78 \times 10^{-5} \mathrm{~kg} /(\mathrm{m} \cdot \mathrm{s})$ and $v_{\mathrm{c}}=340 \mathrm{~m} / \mathrm{s}$, the effect of the pressure variation could be neglected.

\section{The comparisons of theoretical and experimental results}

The total aerodynamic drag of the running train cannot be measured directly because the train is freely levitated above the PMG. The average velocity between check points $A$ and

Table 1 Each fixed parameters in ETSMT

\begin{tabular}{lc}
\hline Parameters & Value $(\mathrm{mm})$ \\
\hline$a_{0}$ & 245 \\
$b_{0}$ & 250 \\
$a_{1}$ & 70 \\
$c$ & 110 \\
$h_{0}$ & 35 \\
$h$ & 10 \\
\hline
\end{tabular}

Table 2 Each experimental parameters in ETSMT

\begin{tabular}{lll}
\hline$b_{r}$ & $a(\mathrm{~mm})$ & $b(\mathrm{~mm})$ \\
\hline 0.10 & 100 & 58.8 \\
0.12 & 100 & 70.6 \\
0.15 & 120 & 73.5 \\
0.18 & 130 & 81.5 \\
0.20 & 140 & 84 \\
\hline
\end{tabular}

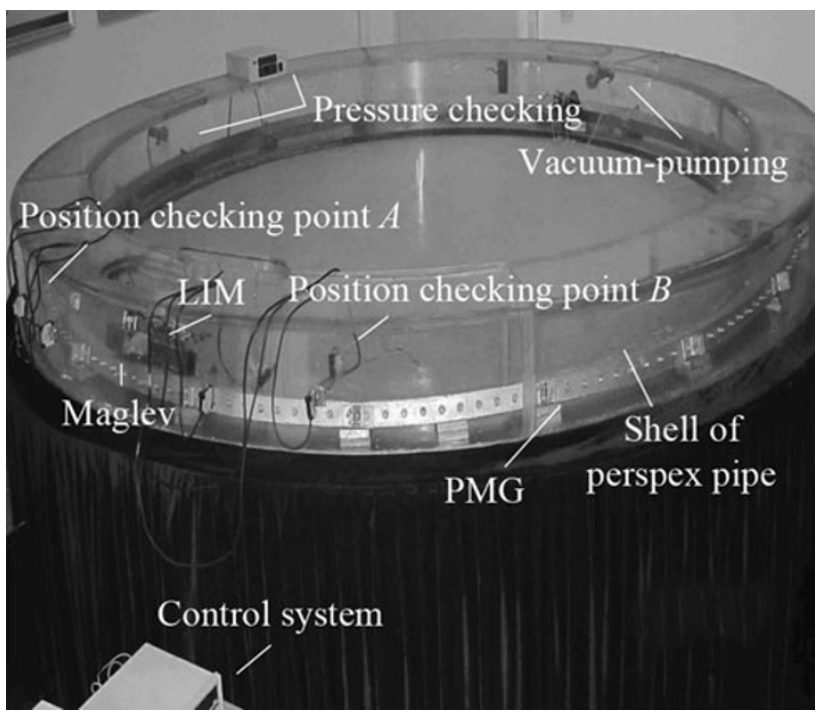

Fig. 7 The experimental system of ETT 


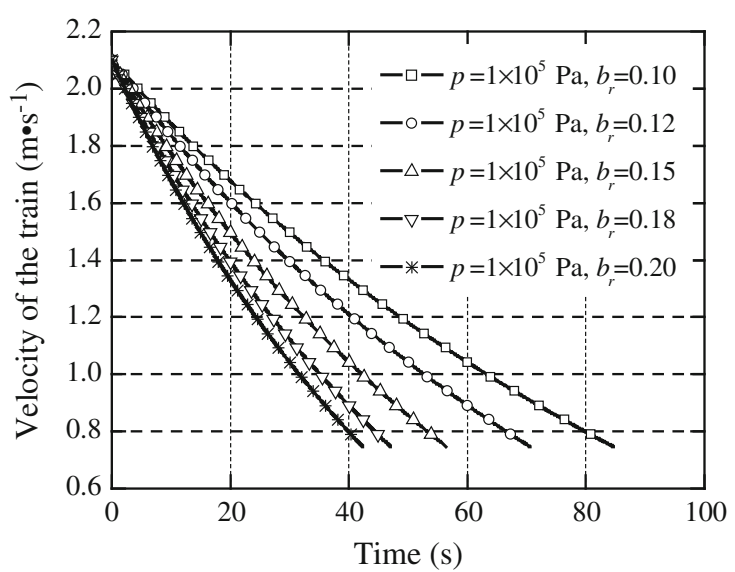

(a) The calculated results

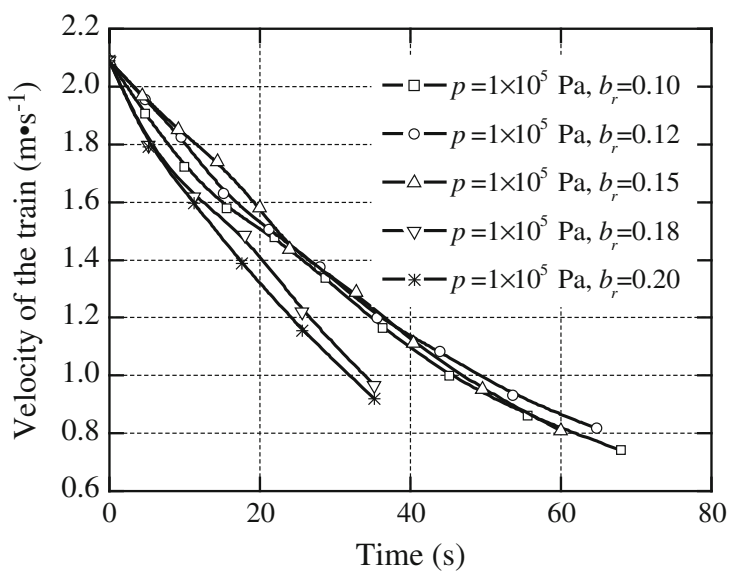

(b) The experimental results

Fig. 8 The relation between the velocity and the time when the pressure is $101,325 \mathrm{~Pa}$

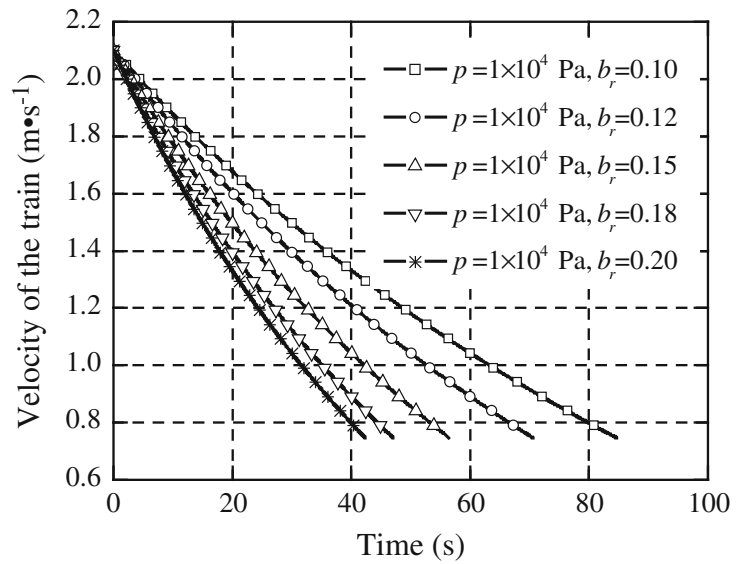

(a) The calculated results

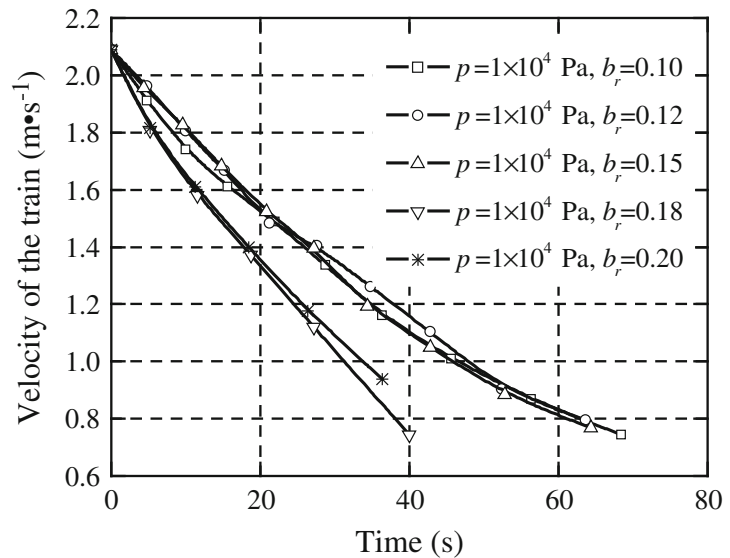

(b) The experimental results

Fig. 9 The relation between the velocity and the time when the pressure is $10,000 \mathrm{~Pa}$

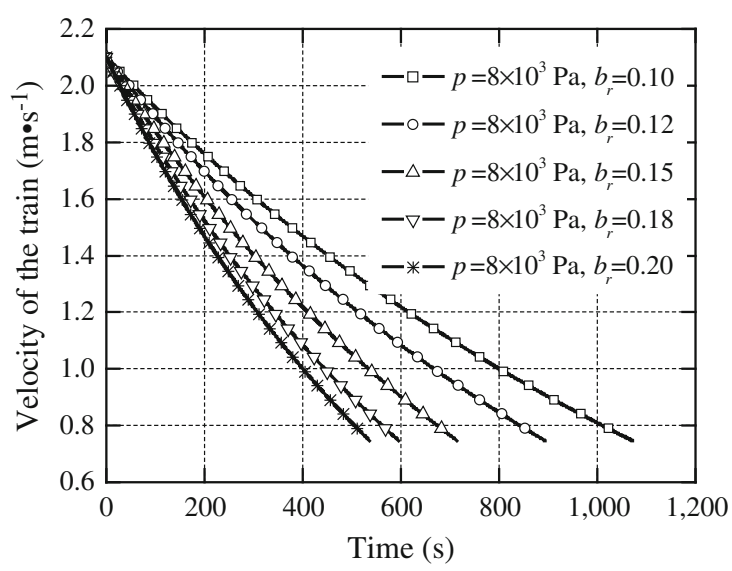

(a) The calculated results

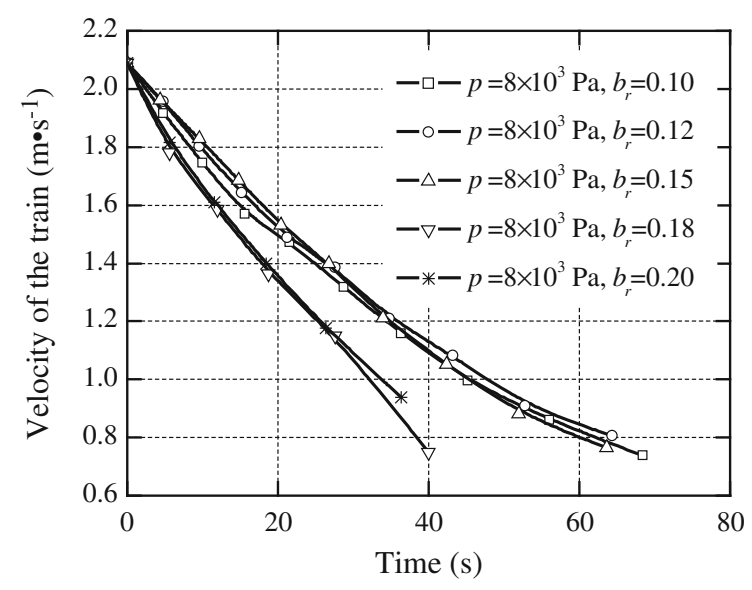

(b) The experimental results

Fig. 10 The relation between the velocity and the time when the pressure is $8,000 \mathrm{~Pa}$ 


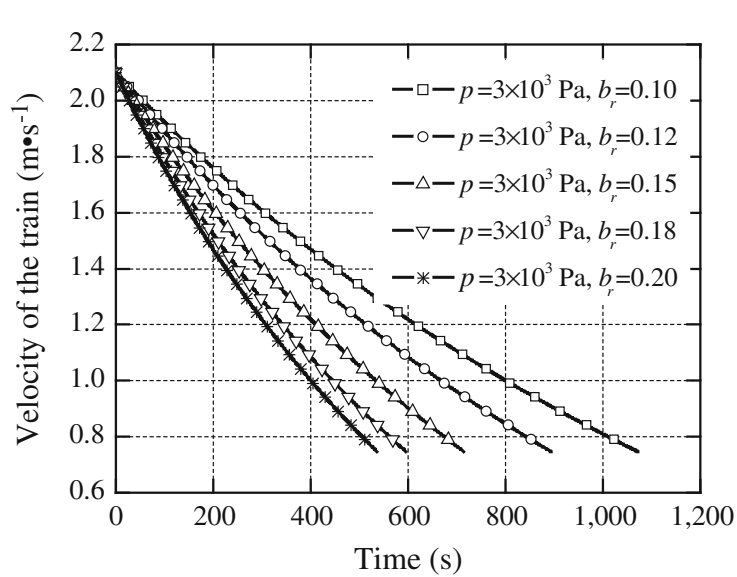

(a) The calculated results

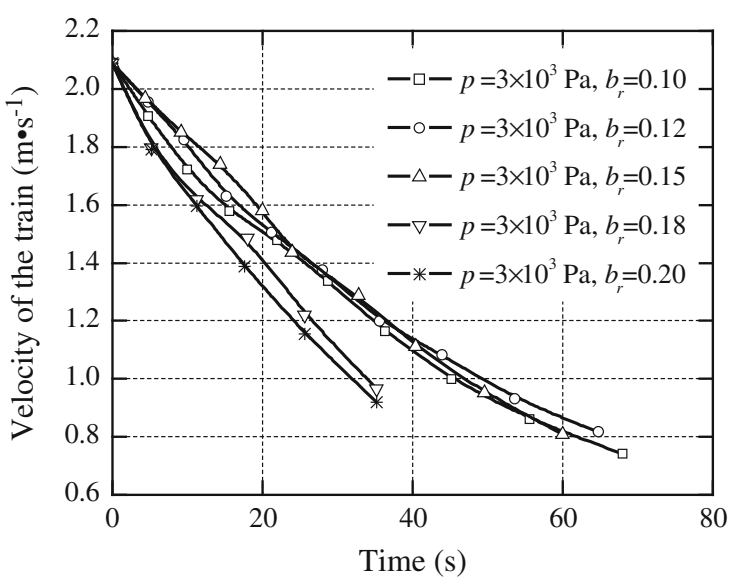

(b) The experimental results

Fig. 11 The relation between the velocity and the time when the pressure is $3,000 \mathrm{~Pa}$

$B$ in Fig. 7 can be calculated by the measured time difference and the length of arc $\overrightarrow{A B}$. The train velocity in experiment was speeded up to $2.2 \mathrm{~m} / \mathrm{s}$ and then the linear motor was stopped. Figure 8a shows the relationship between the decreasing velocity and the time according to Eq. (22), while Fig. 8b shows the experimental result.

When the pressure is constant in the inner tube, the running time is less and the blockage ratio is larger. That is to say, if the blockage ratio is larger, so is the aerodynamic drag. Both the calculated and the experimental results show such a trend.

Figures 9, 10, and 11 illustrate the relation between the velocity and the time under different pressures. We can see if the pressure is decreased, the running time is longer because the negative acceleration is smaller. Let the velocity be $2.2 \mathrm{~m} / \mathrm{s}$ and the blockage rate be 0.2 in Eq. (22). If the pressures are $101,325,10,000,8,000$, and 3,000 $\mathrm{Pa}$, the drags are $0.0,363,0.0,036,0.0,029$, and $0.0,011 \mathrm{~N}$ respectively.

\section{Conclusions}

(1) When the pressure and the blockage ratio are constant, the aerodynamic drag is a quadratic function of the velocity. When the velocity of the train is bigger than the sound velocity, the formula of the aerodynamic drag becomes more complex.

(2) If the blockage ratio is smaller, the drag becomes smaller. In practice, the blockage ratio is impossible to be very small because of the limitation of the pipe's section size. So a suitable blockage ratio should be determined in the design of the ETT system.

(3) If the pressure in the tube is zero, the aerodynamic drag equals to zero no matter how the velocity and the blockage rate vary. This ideal condition is difficult to realize because of the technological limitation.

In this work, the comparison between the theoretical and experimental results was made when the velocity of the Maglev train is small. When the system runs in a lower pressure, more efforts must be made to solve more sophisticated technical problems. Thus, the speed, the pressure, and the blockage ratio each must have reasonable values for ETT. In such a case, the magnetic drag between the Maglev train and PMG may be negligible. In future study, we will consider the effect of magnetic drag between the Maglev train and PMG at high speeds.

Acknowledgments This paper was supported by the National Magnetic Confinement Fusion Science Program (No. 2011GB112001), the Program of International S\&T Cooperation (No. S2013ZR0595), the Fundamental Research Funds for the Central Universities (Nos. SWJTU11ZT16, SWJTU11ZT31), the Science Foundation of Sichuan Province (No. 2011JY0031,2011JY0130).

Open Access This article is distributed under the terms of the Creative Commons Attribution License which permits any use, distribution, and reproduction in any medium, provided the original author(s) and the source are credited.

\section{References}

1. Meins J, Miller L, Mayer WJ (1998) The high speed maglev transportation system transrapid. IEEE Transactions on Magn 24(2):808-811

2. Lee HW, K KC, Lee J (2006) Review of maglev train technologies. IEEE Trans Magn 42(7):1917-1925

3. Shen Z (2001) Dynamic interaction of high speed maglev train on girders and its comparison with the case in ordinary high speed railways. J Traffic Transp Eng 1(1):1-6 (in Chinese)

4. Shen Z (2005) On developing high-speed evacuated tube transportation in China. J Southwest Jiaotong Univ 40(2):133-137 (in Chinese) 
5. Yan L (2006) Progress of the maglev transportation in China. IEEE Trans Appl Supercond 16(2):1138-1141

6. Raghuathan S, Kim HD, Setoguchi T (2002) Aerodynamics of high speed railway train. Prog Aerosp Sci 38(6):469-514

7. Cai YG, Chen SS (1997) Dynamic characteristics of magnetically levitated vehicle systems. Appl Mech Rev, ASME 50(11): $647-670$

8. Wu Q, Yu H, Li H (2004) A study on numerical simulation of aerodynamics for the maglev train. Railw Locomot \& CAR 24(2):18-20 (in Chinese)

9. Xu W, Liao H, Wang W (1998) Study on numerical simulation of aerodynamic drag of train in tunnel. J China Railw Soc 20(2):93-98 (in Chinese)

10. Shu X, Gu C, Liang X et al (2006) The Numerical simulation on the aerodynamic performance of high-speed maglev train with streamlined nose. J Shanghai Jiaotong Univ 40(6):1034-1037 (in Chinese)
11. Zhou X, Zhang D, Zhang Y (2008) Numerical simulation of blockage rate and aerodynamic drag of high-speed train in evacuated tube transportation. Chin J Vacuum Sci Technol 12(28):535-538 (in Chinese)

12. Chen X, Zhao L, MA J, Liu Y (2012) Aerodynamic simulation of evacuated tube maglev trains with different streamlined designs. J Mod Transp 20(2):115-120

13. Jiang J, Bai X, Wu L, Zhang Y (2012) Design consideration of a super-high speed high temperature superconductor maglev evacuated tube transport(I). J Mod Transp 20(2):108-114

14. Fletcher CAJ (1900) Computational Techniques for Fluid Dynamics(Vol.I and II). Springer-Verlag, Berlin

15. Launder BE, Spalding DB (1974) The numberical computation of turbulent flows [J]. Comput Methods Appl Mech Eng 3:269-289

16. Qian Y (2004) Aerodynamics (The first edition). Beihang University Press, Beijing (in Chinese) 